\title{
Some identities for degenerate complete and incomplete $r$-Bell polynomials
}

Jongkyum Kwon', Taekyun Kim², ${ }^{2,}$, Dae San Kim³ and Han Young Kim ${ }^{4}$

"Correspondence: tkkim@kw.ac.kr

${ }^{2}$ School of Science, Xi'an

Technological University, Xi'an, China

${ }^{4}$ Department of Mathematics, Kwangwoon University, Seoul, Republic of Korea

Full list of author information is available at the end of the article

\begin{abstract}
In this paper, we study degenerate complete and incomplete $r$-Bell polynomials. They are generalizations of the recently introduced degenerate $r$-Bell polynomials and degenerate analogues for the complete and incomplete $r$-Bell polynomials. We investigate some properties and identities for these polynomials. In particular, we give explicit formulas for the degenerate complete and incomplete $r$-Bell polynomials.
\end{abstract}

MSC: 11B73; 11B83; 05A19

Keywords: Degenerate complete $r$-Bell polynomials; Degenerate incomplete $r$-Bell polynomials; Extended degenerate complete $r$-Bell polynomials

\section{Introduction}

For any nonzero $\lambda \in \mathbb{R}$, the degenerate exponential function is defined by

$$
e_{\lambda}^{x}(t)=(1+\lambda t)^{\frac{x}{\lambda}}, \quad e_{\lambda}(t)=(1+\lambda t)^{\frac{1}{\lambda}} \quad(\text { see }[1-4]) .
$$

The degenerate Bell polynomials were considered by Kim-Kim-Dolgy in [5] and they are given by

$$
e^{x\left(e_{\lambda}(t)-1\right)}=\sum_{n=0}^{\infty} \operatorname{Bel}_{n}(x \mid \lambda) \frac{t^{n}}{n !} \quad(\text { see }[2])
$$

Note that $\lim _{\lambda \rightarrow 0} \operatorname{Bel}_{n}(x \mid \lambda)=\operatorname{Bel}_{n}(x)(n \geq 0)$, where $\operatorname{Bel}_{n}(x)$ are the ordinary Bell polynomials defined by

$$
e^{x\left(e^{t}-1\right)}=\sum_{n=0}^{\infty} \operatorname{Bel}_{n}(x) \frac{t^{n}}{n !} \quad(\text { see }[6,7])
$$

For $r \in \mathbb{N} \cup\{0\}$, the degenerate $r$-Bell polynomials are given by

$$
e_{\lambda}^{r}(t) e^{x\left(e_{\lambda}(t)-1\right)}=\sum_{n=0}^{\infty} \operatorname{Bel}_{n}^{(r)}(x \mid \lambda) \frac{t^{n}}{n !} \quad(\text { see }[3])
$$

When $x=1, \operatorname{Bel}_{n}^{(r)}(\lambda)=\operatorname{Bel}_{n}^{(r)}(1 \mid \lambda)$ are called the degenerate $r$-Bell numbers.

(c) The Author(s) 2020. This article is licensed under a Creative Commons Attribution 4.0 International License, which permits use, sharing, adaptation, distribution and reproduction in any medium or format, as long as you give appropriate credit to the original author(s) and the source, provide a link to the Creative Commons licence, and indicate if changes were made. The images or other third party material in this article are included in the article's Creative Commons licence, unless indicated otherwise in a credit line to the material. If material is not included in the article's Creative Commons licence and your intended use is not permitted by statutory regulation or exceeds the permitted use, you will need to obtain permission directly from the copyright holder. To view a copy of this licence, visit http://creativecommons.org/licenses/by/4.0/. 
Let $r$ be a nonnegative integer. The $r$-Stirling numbers $S_{2, r}(n, k)$ of the second kind are given by

$$
\frac{1}{k !} e^{r t}\left(e^{t}-1\right)^{k}=\sum_{n=k}^{\infty} S_{2, r}(n+r, k+r) \frac{t^{n}}{n !}
$$

These numbers enumerate the number of partitions of the set $[n]=\{1,2,3, \ldots, n\}$ into $k$ nonempty disjoint subsets in such a way that $1,2,3, \ldots, r$ are in distinct subsets (see [8]). Here we note, in passing, that Simsek [9] introduced the generalized array polynomials which in some special case reduce to the classical array polynomials. The generating function of the array polynomials is given by that in (1.5) with $r$ replaced by the variable $x$. Also, the reader may want to see [10] for some related paper on Stirling numbers.

As a degenerate version of $S_{2}^{(r)}(n+r, k+r)$, the degenerate $r$-Stirling numbers $S_{2, \lambda}^{(r)}(n+$ $r, k+r)$ of the second kind were introduced, and they are defined by

$$
\frac{1}{k !} e_{\lambda}^{r}(t)\left(e_{\lambda}(t)-1\right)^{k}=\sum_{n=k}^{\infty} S_{2, \lambda}^{(r)}(n+r, k+r) \frac{t^{n}}{n !} \quad(\text { see }[3,4,11])
$$

Then it is not difficult to see that (1.6) is equivalent to the following:

$$
(x+r)_{n, \lambda}=\sum_{k=0}^{n} S_{2, \lambda}^{(r)}(n+r, k+r)(x)_{k}
$$

where $(x)_{0, \lambda}=1,(x)_{0}=1,(x)_{n, \lambda}=x(x-\lambda)(x-2 \lambda) \cdots(x-(n-1) \lambda)(n \geq 1)$, and $(x)_{n}=(x)_{n, 1}$ $(n \geq 1)$.

From (1.4) and (1.6), we have

$$
\operatorname{Bel}_{n}^{(r)}(x \mid \lambda)=\sum_{k=0}^{n} S_{2, \lambda}^{(r)}(n+r, k+r) x^{k} \quad(n \geq 0)
$$

The exponential incomplete $r$-Bell polynomials are defined by the generating function (see $[6,12])$

$$
\frac{1}{k !}\left(\sum_{j=1}^{\infty} a_{j} \frac{t^{j}}{j !}\right)^{k}\left(\sum_{i=0}^{\infty} b_{i+1} \frac{t^{i}}{i !}\right)^{r}=\sum_{n=k}^{\infty} B_{n+r, k+r}^{(r)}\left(a_{1}, a_{2}, \ldots ; b_{1}, b_{2}, \ldots\right) \frac{t^{n}}{n !} .
$$

From (1.9), we note that

$$
\begin{aligned}
& B_{n+r, k+r}^{(r)}\left(a_{1}, a_{2}, \ldots ; b_{1}, b_{2}, \ldots\right) \\
& =\sum_{\Lambda(n, k, r)}\left[\frac{n !}{k_{1} ! k_{2} ! k_{3} ! \cdots}\left(\frac{a_{1}}{1 !}\right)^{k_{1}}\left(\frac{a_{2}}{2 !}\right)^{k_{2}}\left(\frac{a_{3}}{3 !}\right)^{k_{3}} \cdots\right] \\
& \quad \times\left[\frac{r !}{r_{0} ! r_{1} ! r_{2} ! \cdots}\left(\frac{b_{1}}{0 !}\right)^{r_{0}}\left(\frac{b_{2}}{1 !}\right)^{r_{1}}\left(\frac{b_{3}}{2 !}\right)^{r_{2}} \cdots\right],
\end{aligned}
$$

where $\Lambda(n, k, r)$ denotes the set of all nonnegative integers $\left(k_{i}\right)_{i \geq 1}$ and $\left(r_{i}\right)_{i \geq 0}$ such that $\sum_{i \geq 1} k_{i}=k, \sum_{i \geq 0} r_{i}=r$ and $\sum_{i \geq 1} i\left(k_{i}+r_{i}\right)=n($ see $[6,12])$. 
Assume that $\left\{a_{i}\right\}_{i \geq 1}$ and $\left\{b_{i}\right\}_{i \geq 1}$ are sequences of positive integers. Then it can be shown $($ see $[6,12])$ that the number $B_{n+r, k+r}^{(r)}\left(a_{1}, a_{2}, \ldots ; b_{1}, b_{2}, \ldots\right)$ counts the number of partitions of a $(n+r)$-set into $(k+r)$ blocks satisfying:

(1) The first $r$ elements belong to different blocks,

(2) Any block of size $i$ containing no elements from the first $r$ elements can be colored with $a_{i}$ colors,

(3) Any block of size $i$ containing one element from the first $r$ elements can be colored with $b_{i}$ colors.

The complete $r$-Bell polynomials are given by

$$
\exp \left(\sum_{i=1}^{\infty} a_{i} \frac{t^{i}}{i !}\right)\left(\sum_{j=0}^{\infty} b_{j+1} \frac{t^{j}}{j !}\right)^{r}=\sum_{n=0}^{\infty} \operatorname{Bel}_{n}^{(r)}\left(a_{1}, a_{2}, \ldots ; b_{1}, b_{2}, \ldots\right) \frac{t^{n}}{n !},
$$

where we note that $\exp (t)=e^{t}$.

Thus, by (1.9) and (1.11), we get

$$
\operatorname{Bel}_{n}^{(r)}\left(a_{1}, a_{2}, \ldots ; b_{1}, b_{2}, \ldots\right)=\sum_{k=0}^{n} B_{n+r, k+r}^{(r)}\left(a_{1}, a_{2}, \ldots ; b_{1}, b_{2}, \ldots\right) .
$$

The degenerate Stirling, Bernoulli, and Euler numbers were introduced by Carlitz as the first degenerate versions of some special numbers. Kim and his colleagues have been studying degenerate versions of many special polynomials and numbers by making use of various different tools. Indeed, they have been investigated by means of generating functions, combinatorial methods, umbral calculus, differential equations, probability theory, and $p$-adic analysis. We should note here that studying degenerate versions of some special polynomials and numbers has been very fruitful and has potential to find many applications in diverse areas. For example, in [4] it is shown that the degenerate $\lambda$-Stirling polynomials of the second kind appear in the expressions of the probability distributions of appropriate random variables. Also, we would like to emphasize that studying degenerate versions is not only applied to polynomials but also extended to transcendental functions. In fact, the degenerate gamma functions were introduced and some interesting results were derived in [1].

Multivariate versions of the Stirling numbers of the second kind and ordinary Bell polynomials are, respectively, the incomplete and complete Bell polynomials. Degenerate versions of the incomplete and complete Bell polynomials are, respectively, the degenerate incomplete and complete Bell polynomials. Extended versions of the degenerate incomplete and complete Bell polynomials are, respectively, the degenerate incomplete and complete $r$-Bell polynomials. We refer the reader to the Introduction in [13] for diverse applications of the complete and incomplete Bell polynomials. In addition, the central complete and incomplete Bell polynomials were treated in the same paper [13]. They are 'central' analogues for the complete and incomplete Bell polynomials and generalizations of the central Bell polynomials and the central factorial numbers of the second kind. We note here, in passing, that the central factorial numbers are as important as the Stirling numbers, though the former received less attention than the latter [14].

In this paper, we will introduce the degenerate incomplete and complete $r$-Bell polynomials, respectively, given in (2.1) and (2.3). We will investigate some properties and 
identities for these polynomials. In particular, we will give some explicit formulas for the degenerate complete and incomplete $r$-Bell polynomials. Lastly, we mention that the extended degenerate $r$-central factorial numbers of the second kind and extended degenerate $r$-central Bell polynomials have been treated in [15].

\section{Degenerate complete and incomplete $r$-Bell polynomials}

Let us consider the following exponential degenerate incomplete $r$-Bell polynomials $B_{n+r, k+r}^{(r)}\left(a_{1}, a_{2}, \ldots ; b_{1}, b_{2}, \ldots \mid \lambda\right)$ given by

$$
\begin{aligned}
B_{n+r, k+r}^{(r)}\left(a_{1}, a_{2}, \ldots ; b_{1}, b_{2}, \ldots \mid \lambda\right) & \\
= & B_{n+r, k+r}^{(r)}\left(a_{1}(1)_{1, \lambda}, a_{2}(1)_{2, \lambda}, \ldots ; b_{1}(1)_{0, \lambda}, b_{2}(1)_{1, \lambda}, \ldots\right) \\
= & \sum_{\Lambda(n, k, r)}\left[\frac{n !}{k_{1} ! k_{2} ! \cdots}\left(\frac{(1)_{1, \lambda} a_{1}}{1 !}\right)^{k_{1}}\left(\frac{(1)_{2, \lambda} a_{2}}{2 !}\right)^{k_{2}} \ldots\right] \\
\quad & \times\left[\frac{r !}{r_{0} ! r_{1} ! \cdots}\left(\frac{(1)_{0, \lambda} b_{1}}{0 !}\right)^{r_{0}}\left(\frac{(1)_{1, \lambda} b_{2}}{1 !}\right)^{r_{1}} \ldots\right],
\end{aligned}
$$

where $n, k \geq 0$, with $n \geq k$.

By (1.9) and (2.1), we also see that

$$
\begin{aligned}
& \frac{1}{k !}\left(\sum_{j=1}^{\infty} a_{j}(1)_{j, \lambda} \frac{t^{j}}{j !}\right)^{k}\left(\sum_{i=0}^{\infty} b_{i+1}(1)_{i, \lambda} \frac{t^{i}}{i !}\right)^{r} \\
& \quad=\sum_{n=k}^{\infty} B_{n+r, k+r}^{(r)}\left(a_{1}(1)_{1, \lambda}, a_{2}(1)_{2, \lambda}, \ldots ; b_{1}(1)_{0, \lambda}, b_{2}(1)_{1, \lambda}, \ldots\right) \frac{t^{n}}{n !} \\
& \quad=\sum_{n=k}^{\infty} B_{n+r, k+r}^{(r)}\left(a_{1}, a_{2}, \ldots ; b_{1}, b_{2}, \ldots \mid \lambda\right) \frac{t^{n}}{n !} .
\end{aligned}
$$

Also, we define the degenerate complete $r$-Bell polynomials by

$$
\operatorname{Bel}_{n}^{(r)}\left(a_{1}, a_{2}, \ldots ; b_{1}, b_{2}, \ldots \mid \lambda\right)=\sum_{k=0}^{n} B_{n+r, k+r}^{(r)}\left(a_{1}, a_{2}, \ldots ; b_{1}, b_{2}, \ldots \mid \lambda\right)
$$

where $\lambda \in \mathbb{R}$ and $n \in \mathbb{N} \cup\{0\}$.

Note that

$$
\lim _{\lambda \rightarrow 0} \operatorname{Bel}_{n}^{(r)}\left(a_{1}, a_{2}, \ldots ; b_{1}, b_{2}, \ldots \mid \lambda\right)=\operatorname{Bel}_{n}^{(r)}\left(a_{1}, a_{2}, \ldots ; b_{1}, b_{2}, \ldots\right) \quad(n \geq 0) .
$$

In addition, the extended degenerate complete r-Bell polynomials are defined by the generating function

$$
\begin{gathered}
\exp \left(u \sum_{j=1}^{\infty} a_{j}(1)_{j, \lambda} \frac{t^{j}}{j !}\right)\left(\sum_{i=0}^{\infty} b_{i+1}(1)_{i, \lambda} \frac{t^{i}}{i !}\right)^{r} \\
=\sum_{n=0}^{\infty} \operatorname{Bel}_{n}^{(r)}\left(u\left|a_{1}, a_{2}, \ldots ; b_{1}, b_{2}, \ldots\right| \lambda\right) \frac{t^{n}}{n !} .
\end{gathered}
$$


From (2.2), we note that

$$
\begin{aligned}
& \sum_{n=0}^{\infty} \operatorname{Bel}_{n}^{(r)}\left(u\left|a_{1}, a_{2}, \ldots ; b_{1}, b_{2}, \ldots\right| \lambda\right) \frac{t^{n}}{n !} \\
& \quad=\sum_{k=0}^{\infty} u^{k} \frac{1}{k !}\left(\sum_{j=1}^{\infty} a_{j}(1)_{j, \lambda} \frac{t^{j}}{j !}\right)^{k}\left(\sum_{i=0}^{\infty} b_{i+1}(1)_{i, \lambda} \frac{t^{i}}{i !}\right)^{r} \\
& \quad=\sum_{k=0}^{\infty} u^{k} \sum_{n=k}^{\infty} B_{n+r, k+r}^{(r)}\left(a_{1}, a_{2}, \ldots ; b_{1}, b_{2}, \ldots \mid \lambda\right) \frac{t^{n}}{n !} \\
& \quad=\sum_{n=0}^{\infty} \sum_{k=0}^{n} u^{k} B_{n+r, k+r}^{(r)}\left(a_{1}, a_{2}, \ldots ; b_{1}, b_{2}, \ldots \mid \lambda\right) \frac{t^{n}}{n !} .
\end{aligned}
$$

By (2.5), we obtain the following theorem.

Theorem 2.1 For $n, r \geq 0$, we have

$$
\operatorname{Bel}_{n}^{(r)}\left(u\left|a_{1}, a_{2}, \ldots ; b_{1}, b_{2}, \ldots\right| \lambda\right)=\sum_{k=0}^{n} u^{k} B_{n+r, k+r}^{(r)}\left(a_{1}, a_{2}, \ldots ; b_{1}, b_{2}, \ldots \mid \lambda\right)
$$

By (2.5) and (2.3), we get

$$
\operatorname{Bel}_{n}^{(r)}\left(1\left|a_{1}, a_{2}, \ldots ; b_{1}, b_{2}, \ldots\right| \lambda\right)=\operatorname{Bel}_{n}^{(r)}\left(a_{1}, a_{2}, \ldots ; b_{1}, b_{2}, \ldots \mid \lambda\right) .
$$

We now observe that

$$
\begin{aligned}
\exp & \left(\sum_{i=1}^{\infty} x_{i}(1)_{i, \lambda} \frac{t^{i}}{i !}\right) \\
= & 1+\sum_{k=1}^{\infty}\left(\sum_{i=1}^{\infty} x_{i}(1)_{i, \lambda} \frac{t^{i}}{i !}\right)^{k} \frac{1}{k !} \\
= & 1+\frac{1}{1 !} \sum_{i=1}^{\infty} x_{i}(1)_{i, \lambda} \frac{t^{i}}{i !}+\frac{1}{2 !}\left(\sum_{i=1}^{\infty} x_{i}(1)_{i, \lambda} \frac{t^{i}}{i !}\right)^{2}+\frac{1}{3 !}\left(\sum_{i=1}^{\infty} x_{i}(1)_{i, \lambda} \frac{t^{i}}{i !}\right)^{3}+\cdots \\
= & 1+\frac{x_{1}}{1 !}(1)_{1, \lambda} t+\left(\frac{x_{2}}{2 !}(1)_{2, \lambda}+\frac{1}{2 !} x_{1}^{2}(1)_{1, \lambda}^{2}\right)^{2} \\
& +\left(\frac{x_{3}}{3 !}(1)_{3, \lambda}+\frac{x_{1} x_{2}}{2 !}(1)_{1, \lambda}(1)_{2, \lambda}+\frac{x_{1}^{3}}{3 !}(1)_{1, \lambda}^{3}\right)^{3}+\cdots \\
= & \sum_{k=0}^{\infty} \sum_{m_{1}+2 m_{2}+\cdots+k m_{k}=k} \frac{k !}{m_{1} ! m_{2} ! \cdots m_{k} !}\left(\frac{x_{1}}{1 !}(1)_{1, \lambda}\right)^{m_{1}}\left(\frac{x_{2}}{2 !}(1)_{2, \lambda}\right)^{m_{2}} \\
& \times \cdots \times\left(\frac{x_{k}}{k !}(1)_{k, \lambda}\right)^{m_{k}} \frac{t^{k}}{k !},
\end{aligned}
$$

and

$$
\left(\sum_{j=0}^{\infty}(1)_{j, \lambda} b_{j+1} \frac{t^{j}}{j !}\right)^{r}=\sum_{m=0}^{\infty} \sum_{l_{1}+\cdots+l_{r}=m} \frac{m !}{l_{1} ! \cdots l_{r} !}\left(\prod_{i=1}^{r}(1)_{l_{i}, \lambda} b_{l_{i}+1}\right) \frac{t^{m}}{m !} .
$$


From (2.8) and (2.9), we obtain the following theorem.

Theorem 2.2 For $k \geq 0$ and $\lambda \in \mathbb{R}$, we have

$$
\begin{aligned}
\exp & \left(\sum_{i=1}^{\infty} x_{i}(1)_{i, \lambda} \frac{t^{i}}{i !}\right)\left(\sum_{j=0}^{\infty}(1)_{j, \lambda} b_{j+1} \frac{t^{j}}{j !}\right)^{r} \\
= & \sum_{n=0}^{\infty} \sum_{k=0}^{n}\left(\begin{array}{l}
n \\
k
\end{array}\right)_{m_{1}+2 m_{2}+\cdots+k m_{k}=k} \sum_{l_{1}+\cdots+l_{r}=n-k} \frac{k !}{m_{1} ! m_{2} ! \cdots m_{k} !} \\
& \times\left(\frac{x_{1}}{1 !}(1)_{1, \lambda}\right)^{m_{1}}\left(\frac{x_{2}}{2 !}(1)_{2, \lambda}\right)^{m_{2}} \times \cdots \times\left(\frac{x_{k}}{k !}(1)_{k, \lambda}\right)^{m_{k}} \\
& \times \frac{(n-k) !}{l_{1} ! \cdots l_{r} !}\left(\prod_{i=1}^{r}(1)_{l_{i}, \lambda} b_{l_{i}+1}\right) \frac{t^{n}}{n !} .
\end{aligned}
$$

From (2.4) and (2.7), we note that

$$
\begin{gathered}
\exp \left(\sum_{j=1}^{\infty} x_{j}(1)_{j, \lambda} \frac{t^{j}}{j !}\right)\left(\sum_{i=0}^{\infty} b_{i+1}(1)_{i, \lambda} \frac{t^{i}}{i !}\right)^{r} \\
=\sum_{n=0}^{\infty} \operatorname{Bel}_{n}^{(r)}\left(x_{1}, x_{2}, \ldots ; b_{1}, b_{2}, \ldots \mid \lambda\right) \frac{t^{n}}{n !} .
\end{gathered}
$$

Using (2.10) and (2.11), we get

$$
\begin{aligned}
\operatorname{Bel}_{n}^{(r)}\left(x_{1}, x_{2}, \ldots ; b_{1}, b_{2}, \ldots \mid \lambda\right) & =\sum_{k=0}^{n}\left(\begin{array}{l}
n \\
k
\end{array}\right) \sum_{m_{1}+2 m_{2}+\cdots+k m_{k}=k} \sum_{l_{1}+\cdots+l_{r}=n-k} \frac{k !}{m_{1} ! m_{2} ! \cdots m_{k} !} \\
& \times\left(\frac{x_{1}}{1 !}(1)_{1, \lambda}\right)^{m_{1}}\left(\frac{x_{2}}{2 !}(1)_{2, \lambda}\right)^{m_{2}} \times \cdots \times\left(\frac{x_{k}}{k !}(1)_{k, \lambda}\right)^{m_{k}} \\
& \times \frac{(n-k) !}{l_{1} ! \cdots l_{r} !} \prod_{i=1}^{r}(1)_{l_{i}, \lambda} b_{l_{i}+1} .
\end{aligned}
$$

From (2.1), (2.4), and (2.6), we note that

$$
\begin{aligned}
& \exp \left(x \sum_{j=1}^{\infty}(1)_{j, \lambda} \frac{t^{j}}{j !}\right)\left(\sum_{i=0}^{\infty}(1)_{i, \lambda} \frac{t^{i}}{i !}\right)^{r} \\
& =\sum_{n=0}^{\infty} \sum_{k=0}^{n} x^{k} B_{n+r, k+r}^{(r)}\left((1)_{1, \lambda},(1)_{2, \lambda}, \ldots ;(1)_{0, \lambda},(1)_{1, \lambda}, \ldots\right) \frac{t^{n}}{n !} .
\end{aligned}
$$

On the other hand,

$$
\exp \left(x \sum_{j=1}^{\infty}(1)_{j, \lambda} \frac{t^{j}}{j !}\right)\left(\sum_{i=0}^{\infty}(1)_{i, \lambda} \frac{t^{i}}{i !}\right)^{r}
$$




$$
\begin{aligned}
& =\exp \left(x \sum_{j=1}^{\infty}\left(\frac{1}{\lambda}\right)_{j} \frac{(\lambda t)^{j}}{j !}\right)\left(\sum_{i=0}^{\infty}\left(\frac{1}{\lambda}\right)_{i} \frac{(\lambda t)^{i}}{i !}\right)^{r} \\
& =e^{x\left(e_{\lambda}(t)-1\right)} e_{\lambda}^{r}(t) \\
& =\sum_{n=0}^{\infty} \operatorname{Bel}_{n}^{(r)}(x \mid \lambda) \frac{t^{n}}{n !} .
\end{aligned}
$$

Therefore, by (2.13) and (2.14), we get

$$
\sum_{k=0}^{n} x^{k} B_{n+r, k+r}^{(r)}\left((1)_{1, \lambda},(1)_{2, \lambda}, \ldots ;(1)_{0, \lambda},(1)_{1, \lambda}, \ldots\right)=\mathrm{Bel}_{n}^{(r)}(x \mid \lambda) .
$$

Thus, by (2.11), (2.13) and (2.14), we obtain the following theorem.

Theorem 2.3 For $n, k, r \geq 0$ and $\lambda \in \mathbb{R}$, we have

$$
\begin{aligned}
B_{n}^{(r)} & (x, \ldots, x ; 1,1, \ldots \mid \lambda) \\
& =\sum_{k=0}^{n} x^{k} B_{n+r, k+r}^{(r)}\left((1)_{1, \lambda},(1)_{2, \lambda}, \ldots ;(1)_{0, \lambda},(1)_{1, \lambda}, \ldots\right) \\
& =\operatorname{Bel}_{n}^{(r)}(x \mid \lambda) .
\end{aligned}
$$

From (2.2) and (1.6), we have

$$
\begin{aligned}
\sum_{n=k}^{\infty} B_{n+r, k+r}^{(r)}\left((1)_{1, \lambda},(1)_{2, \lambda}, \ldots ;(1)_{0, \lambda},(1)_{1, \lambda}, \ldots\right) \frac{t^{n}}{n !} \\
=\frac{1}{k !}\left(\sum_{j=1}^{\infty}(1)_{j, \lambda} \frac{t^{j}}{j !}\right)^{k}\left(\sum_{i=0}^{\infty}(1)_{i, \lambda} \frac{t^{i}}{i !}\right)^{r} \\
=\frac{1}{k !}\left(e_{\lambda}(t)-1\right)^{k} e_{\lambda}^{r}(t)=\sum_{n=k}^{\infty} S_{2, \lambda}^{(r)}(n+r, k+r) \frac{t^{n}}{n !} \quad(k \geq 0) .
\end{aligned}
$$

Comparing the coefficients on both sides of (2.17), we obtain the following theorem.

Theorem 2.4 For $n, k, r \geq 0$ and $\lambda \in \mathbb{R}$, we have

$$
\begin{aligned}
S_{2, \lambda}^{(r)}(n+r, k+r)= & B_{n+r, k+r}^{(r)}\left((1)_{1, \lambda},(1)_{2, \lambda}, \ldots ;(1)_{0, \lambda},(1)_{1, \lambda}, \ldots\right) \\
= & \sum_{\Lambda(n, k, r)}\left[\frac{n !}{k_{1} ! k_{2} ! \cdots}\left(\frac{(1)_{1, \lambda}}{1 !}\right)^{k_{1}}\left(\frac{(1)_{2, \lambda}}{2 !}\right)^{k_{2}} \cdots\right] \\
& \times\left[\frac{r !}{r_{0} ! r_{1} ! \cdots}\left(\frac{(1)_{0, \lambda}}{0 !}\right)^{r_{0}}\left(\frac{(1)_{1, \lambda}}{1 !}\right)^{r_{1}} \cdots\right] .
\end{aligned}
$$

Now, we observe that

$$
\sum_{n=0}^{\infty} \operatorname{Bel}_{n}^{(r)}(x \mid \lambda) \frac{t^{n}}{n !}=e^{x\left(e_{\lambda}(t)-1\right)} e_{\lambda}^{r}(t)
$$




$$
\begin{aligned}
& =e^{-x} e^{x e_{\lambda}(t)} e_{\lambda}^{r}(t)=e^{-x} \sum_{k=0}^{\infty} \frac{x^{k}}{k !} e_{\lambda}^{k+r}(t) \\
& =e^{-x} \sum_{k=0}^{\infty} \frac{x^{k}}{k !} \sum_{n=0}^{\infty}(k+r)_{n, \lambda} \frac{t^{n}}{n !}=\sum_{n=0}^{\infty}\left(e^{-x} \sum_{k=0}^{\infty} \frac{(k+r)_{n, \lambda}}{k !} x^{k}\right) \frac{t^{n}}{n !} .
\end{aligned}
$$

From (2.19), we obtain the following Dobinski-type formula for the degenerate $r$-Bell polynomials.

Theorem 2.5 For $n, r \geq 0$ and $\lambda \in \mathbb{R}$, we have

$$
\operatorname{Bel}_{n}^{(r)}(x \mid \lambda)=\frac{1}{e^{x}} \sum_{k=0}^{\infty} \frac{(k+r)_{n, \lambda}}{k !} x^{k}
$$

\section{Conclusions}

There are various means of studying special polynomials and numbers, for example, generating functions, combinatorial methods, umbral calculus, $p$-adic analysis, differential equations, probability, orthogonal polynomials, and special functions, to cite a few $[7,14,16]$. These ways of investigating special polynomials and numbers can be applied also to degenerate versions of such polynomials and numbers. Indeed, in recent years, many mathematicians have drawn their attention to studies of degenerate versions of quite a few special polynomials and numbers by making use of the aforementioned methods $[2,5,11,17]$. Also, it is worth mentioning that one can study degenerate versions not only of special polynomials but also of transcendental functions like gamma functions [1].

Here, we introduced the degenerate incomplete and complete $r$-Bell polynomials and studied properties and identities of these polynomials. Indeed, we expressed the extended degenerate complete $r$-Bell polynomials in terms of the degenerate incomplete $r$-Bell polynomials. We also obtained an explicit expression for the complete $r$-Bell polynomials. We noted that the degenerate $r$-Bell polynomials $\operatorname{Bel}_{n}^{(r)}(x \mid \lambda)$ can be represented in terms of the degenerate incomplete $r$-Bell polynomials. Likewise, it was shown that the degenerate $r$-Stirling numbers of the second kind $S_{2, \lambda}^{(r)}(n+r, k+r)$ can be expressed in terms of the degenerate incomplete $r$-Bell polynomials. Further, we obtained a Dobinski-type formula for the degenerate $r$-Bell polynomials.

As one of our future projects, we would like to continue pursuing this line of research, namely, by studying degenerate versions of some special polynomials and numbers, find their applications in mathematics, physics, and engineering.

\section{Acknowledgements}

The authors would like to thank the referees for their helpful comments and suggestions.

Funding

This work was supported by the National Research Foundation of Korea (NRF) grant funded by the Korea government (MEST) (No. 2017R1E1A1A03070882).

Availability of data and materials

Not applicable.

Competing interests

The authors declare that they have no competing interests. 
Authors' contributions

TK and DSK conceived of the framework and structured the whole paper; TK wrote the paper; JK paid for the article-processing charge of the paper; HYK typed the paper; DSK and TK completed the revision of the article. All authors read and approved the final manuscript.

\section{Author details}

${ }^{1}$ Department of Mathematics Education and ERI, Gyeongsang National University, Jinju, Republic of Korea. ${ }^{2}$ School of Science, Xi'an Technological University, Xi'an, China. ${ }^{3}$ Department of Mathematics, Sogang University, Seoul, Republic of Korea. ${ }^{4}$ Department of Mathematics, Kwangwoon University, Seoul, Republic of Korea.

\section{Publisher's Note}

Springer Nature remains neutral with regard to jurisdictional claims in published maps and institutional affiliations.

Received: 8 November 2019 Accepted: 23 January 2020 Published online: 30 January 2020

\section{References}

1. Kim, T., Kim, D.S.: Degenerate Laplace transform and degenerate gamma function. Russ. J. Math. Phys. 23(2), 241-248 (2017)

2. Kim, T., Kim, D.S., Dolgy, D.V.: On partially degenerate Bell numbers and polynomials. Proc. Jangjeon Math. Soc. 20(3), 337-345 (2017)

3. Kim, T., Kim, D.S., Jang, G.-W.: Extended Stirling polynomials of the second kind and extended Bell polynomials. Proc. Jangjeon Math. Soc. 20(3), 365-376 (2017)

4. Kim, T., Yao, Y., Kim, D.S., Jang, G.-W.: Degenerate $r$-Stirling numbers and r-Bell polynomials. Russ. J. Math. Phys. 25(1), 44-58 (2018)

5. Kim, T., Kim, D.S.: On degenerate Bell numbers and polynomials. Rev. R. Acad. Cienc. Exactas Fís. Nat., Ser. A Mat. $111(2), 435-446$ (2017)

6. Carlitz, L.: Some remarks on the Bell numbers. Fibonacci Q. 18(1), 66-73 (1990)

7. Comtet, L.: Advanced Combinatorics: The Art of Finite and Infinite Expansions. Reidel, Dordrecht (1974)

8. Broder, A.Z.: The $r$-Stirling numbers. Discrete Math. 49(3), 241-259 (1984)

9. Simsek, Y.: Generating functions for generalized Stirling type numbers, array type polynomials, Eulerian type polynomials and their applications. Fixed Point Theory Appl. 2013, 87 (2013)

10. Simsek, Y.: On q-deformed Stirling numbers. Int. J. Math. Comput. 15(2), 70-80 (2012)

11. Kim, T.: A note on degenerate Stirling polynomials of the second kind. Proc. Jangjeon Math. Soc. 20(3), 319-331 (2017)

12. Simsek, Y.: Identities and relations related to combinatorial numbers and polynomials. Proc. Jangjeon Math. Soc 20(1), 127-135 (2017)

13. Kim, T., Kim, D.S., Jang, G.-W.: On central complete and incomplete Bell polynomials I. Symmetry 11(2), Article ID 288 (2019)

14. Butzer, P.L., Schmidt, M., Stark, E.L., Vogt, L.: Central factorial numbers; their main properties and some applications. Numer. Funct. Anal. Optim. 10(5-6), 419-488 (1989)

15. Kim, D.S., Dolgy, D.V., Kim, T., Kim, D.: Extended degenerate r-central factorial numbers of the second kind and extended degenerate r-central Bell polynomials. Symmetry 11(4), Article ID 595 (2019)

16. Kim, T., Kim, D.S., Kim, H.Y., Kwon, J.: Degenerate Stirling polynomials of the second kind and some applications. Symmetry 11(8), Article ID 1046 (2019)

17. Kim, T., Kim, D.S.: Degenerate central Bell numbers and polynomials. Rev. R. Acad. Cienc. Exactas Fís. Nat., Ser. A Mat. (2019). https://doi.org/10.1007/s13398-019-00637-0

\section{Submit your manuscript to a SpringerOpen ${ }^{\circ}$ journal and benefit from:}

- Convenient online submission

- Rigorous peer review

- Open access: articles freely available online

- High visibility within the field

- Retaining the copyright to your article

Submit your next manuscript at $\gg$ springeropen.com 